\title{
Spreading behavior of an impacting drop on a structured rough surface
}

\author{
D. Sivakumara) \\ Department of Aerospace Engineering, Indian Institute of Science, Bangalore 560012, India \\ K. Katagiri, T. Sato, and H. Nishiyama \\ Electromagnetic Intelligent Fluids Laboratory, Institute of Fluid Science, Tohoku University, \\ 2-1-1 Katahira, Aoba-ku, Sendai-shi, Miyagi 980-8577, Japan
}

(Received 8 November 2004; accepted 6 July 2005; published online 3 October 2005)

\begin{abstract}
The spreading of water drops impinging on structured rough surfaces is studied experimentally. The rough surfaces are specially prepared with a regular pattern of surface asperities. The arrangement of the square-shaped surface asperities creates channel-like grooves on the surface. A video microscope along with a controlled light exposure system is used to construct the image sequences of the spreading process. The images are digitally analyzed to measure the temporal variation of the spreading drop diameter $2 R$. Results are obtained for three rough surfaces with varying asperity heights in the range of 100-500 $\mu \mathrm{m}$ and for different impact drop conditions with Weber number We in the range of 35-225. The results on the temporal variation of $2 R$ show that, on the structured rough surfaces, the spreading occurs simultaneously both inside and above the texture pattern of the surfaces. For a given surface geometry, the volume of liquid flowing inside the grooves of the surface increases with increasing We. Consequently, the values of $2 R$ measured inside the texture pattern are larger than those measured above the texture pattern, and their difference increases with increasing We. The arrangement of the surface asperities influences the spreading pattern of an impacting drop spreading axisymmetrically. For the texture geometry used in the present study, the spreading pattern resembles a regular rhombus shape for the impact of low We drops and becomes complex at high We. The spreading distances, measured both inside and above the texture pattern of the structured rough surfaces, are nearer to the measurements recorded on the smooth surface if the asperity height of the rough surface is smaller than the thickness of the spreading liquid lamella; however, the surface asperities influence the spreading pattern drastically and create a liquid splash. (C) 2005 American Institute of Physics. [DOI: 10.1063/1.2033627]
\end{abstract}

\section{INTRODUCTION}

The interactive process between a liquid drop and a solid surface is of interest in the impact of liquid drops on dry solid surfaces. This is observed in several practical applications such as liquid and metal printing technologies, microfabrication processes involving molten metal droplets, thinfilm coating on solid surfaces, and formation of uniform solder bumps on integrated circuits. ${ }^{1-6}$ The typical size of liquid drops used in these applications is of the order of tens of microns, and hence the role of the surface features of the solid surface, for instance, the asperities and microgrooves characterizing the roughness pattern of the solid surface, on the drop impact process cannot be ignored. An attempt has been made in this study to investigate the role of surface roughness on the transport of a spreading liquid droplet during the impact process by conducting experiments of drop impact on specially prepared rough surfaces. The rough surfaces encompass square-shaped asperities and grooves of rectangular cross section constructed in a specific pattern.

The drop impact phenomenon has been studied for more than a century. Upon impact of a liquid drop on a dry solid surface, the liquid drop spreads out axisymmetrically from

\footnotetext{
a)Telephone: 91 (0)80 2293 3022. Fax: 91 (0)80 2360 0134. Electronic mail: dskumar@aero.iisc.ernet.in
}

the impact point, reaches a maximum spread diameter and subsequently retracts towards the impact point. Several recently published articles on this topic ${ }^{7-13}$ have reviewed the current level of understanding on the various physical aspects involved in the impact process. Particularly, the review article by Rein ${ }^{7}$ provides a detailed review of these phenomena. Many of the complexities involved in the drop impact process, particularly on a smooth surface, can be simulated by using currently available numerical models. ${ }^{8-13}$ The major physical parameters that influence the drop impact process are the size and velocity of the drop prior to the impact, the liquid properties of the drop, and the surface characteristics of the solid surface. The drop parameters are grouped in terms of nondimensional numbers such as the Weber number We (the ratio of inertial to surface tension forces) and the Ohnesorge number Oh (the ratio of viscous to surface tension forces). Schiaffino and Sonin ${ }^{10}$ provide a brief summary of the impact characteristics for different combinations of We and $\mathrm{Oh}$. The surface characteristics, mainly the wettability and mean roughness, are shown to influence the impact process significantly. ${ }^{14-19}$ The wettability of a solid surface can be described by the contact angle of the spreading liquid with the solid surface through the well-known Young's relation. ${ }^{20}$ Under a microscopic view, it depends on the chemical composition of the solid surface in contact with the liquid as well as on the surface irregularities. Thus, for a 


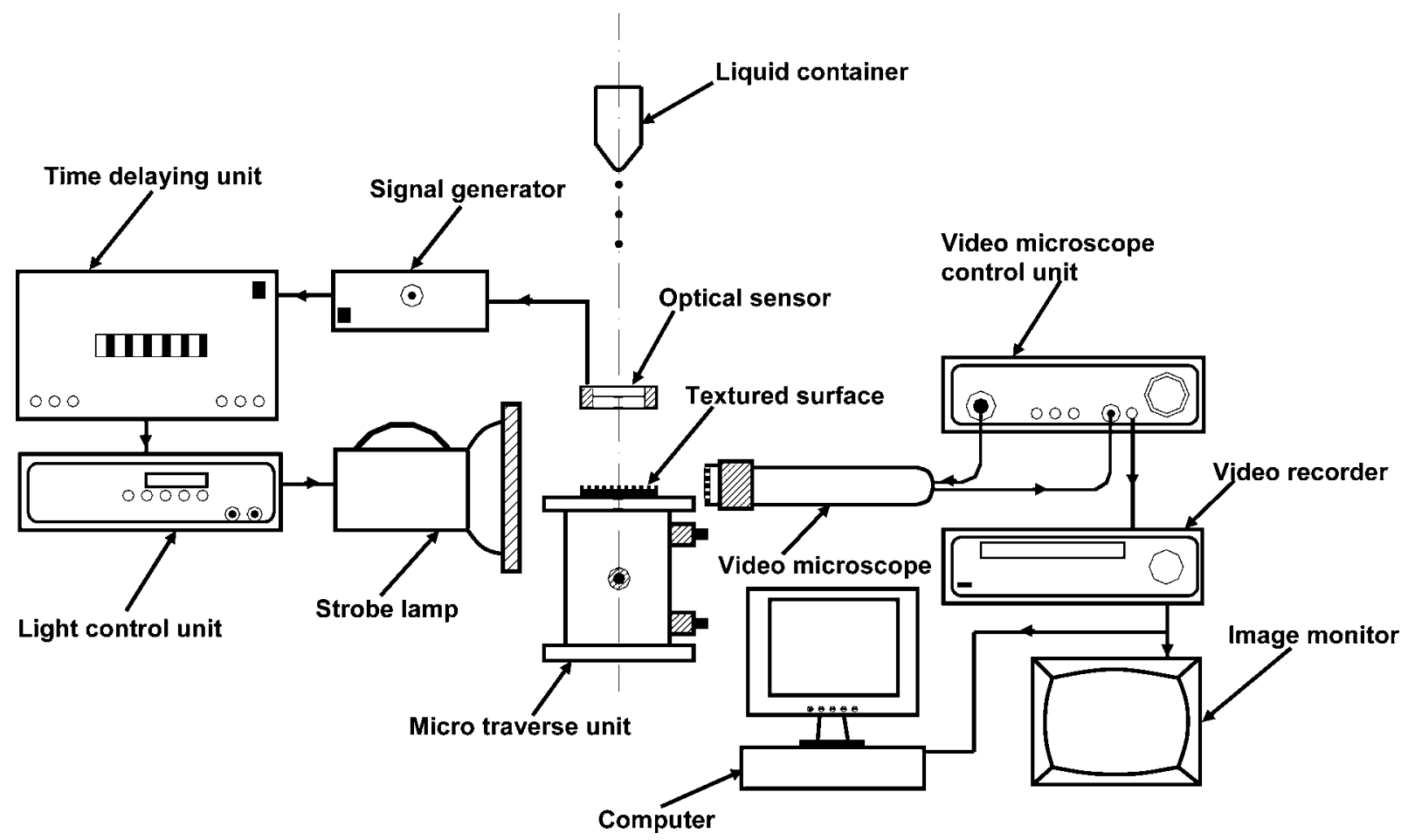

FIG. 1. Schematic diagram of the experimental apparatus.

given liquid droplet, the material properties of the solid surface influence the wettability behavior and hence the spreading process. Several previous experimental studies on drop impact have reported the measurements of contact angle for different smooth surfaces. ${ }^{12,13,19}$

The impacting drop exhibits a splash, identified by the ejection to tiny droplets from the periphery of the spreading drop in the regimes of high inertial forces. For a given liquid droplet and target surface conditions, the impacting drop splashes at a specific combination of We and Oh. Previous studies ${ }^{17}$ cited a physical parameter $K$ in terms of We and Oh to describe the splash behavior, and the value of $K$, at which the splash is first seen, is denoted as the splashing limit $K_{\text {crit }}$. Stow and Hadfield ${ }^{14}$ studied the splash behavior of impacting water drops on rough aluminum surfaces and showed that the splashing limit of an impacting liquid drop decreases with increasing mean surface roughness $R_{a}$. A later study by Range and Feuillebois ${ }^{15}$ arrived at a similar conclusion based on the experimental data collected on rough surfaces with different materials of varying $R_{a}$. They also studied the flow dynamics of the liquid lamella developing from the impact and observed that the number of undulations responsible for the creation of finger-like liquid jets at the periphery of the liquid lamella decreases with increasing $R_{a}$. In other words, the number of fingers counted at the periphery of the spreading drop decreases with increasing $R_{a}$. In a similar study on the impact of molten tin drops on rough stainless steel surfaces, Shakeri and Chandra ${ }^{16}$ observed the same behavior on the number of fingers versus $R_{a}$.

The rough surfaces used in the above-mentioned studies ${ }^{14-19}$ consist of microasperities of different shapes and sizes distributed randomly over the surfaces. Although the studies are very helpful in identifying the complexities caused by a rough surface pattern on the spreading process of an impacting drop, the reported results lack the physical details on the spreading of liquid inside the microgrooves and between the microasperities of the rough surfaces. However, it is evident from literature that the surface asperities may influence several physical processes involved in the impact phenomenon. These processes may include the movement of the liquid contact line associated with surface wetting, ${ }^{21,22}$ the spreading of the liquid inside the grooves of the surface, ${ }^{23}$ and the effect of the asperity shape on the liquid spreading. ${ }^{24}$ In this experimental work, we study the impact of water drops on structured rough surfaces, specially prepared solid substrates encompassing tiny asperities arranged in a specific pattern, and undertake to understand the details of the spreading process of liquid drops on structured rough surfaces with different impact conditions. The results obtained from these experiments are also helpful for the analysis of the drop impact process on rough surfaces encountered in practical applications. Similar structured rough surfaces have been used in several recent studies, however, mainly to investigate the wetting behavior of rough surfaces. ${ }^{24-27}$ Note that the term textured substrate is inadvertently used in this paper instead of structured rough surface. The geometrical details of the textured substrates used in the present study are given in Sec. II along with the salient features of the experimental apparatus. The experimental results obtained from this study are discussed in Sec. III. In Sec. IV, the major conclusions obtained from this investigation are listed.

\section{EXPERIMENTAL DETAILS}

A diagram of the experimental apparatus used in the present study is shown in Fig. 1. The impact process of an 
TABLE I. Mean and standard deviations of the droplet size for different experiments.

\begin{tabular}{cccccc}
\hline \hline $\begin{array}{c}H \\
(\mathrm{~cm})\end{array}$ & $\begin{array}{c}\text { Mean } D_{o, x} \\
(\mathrm{~mm})\end{array}$ & $\begin{array}{c}\text { Mean } D_{o, y} \\
(\mathrm{~mm})\end{array}$ & $S D_{x} / D_{o, x}$ & $S D_{y} / D_{o, y}$ & $D_{o, x} / D_{o, y}$ \\
\hline 5.0 & 3.11 & 3.02 & 0.012 & 0.007 & 1.03 \\
7.5 & 3.14 & 2.97 & 0.009 & 0.007 & 1.06 \\
10.0 & 3.07 & 3.10 & 0.033 & 0.019 & 0.99 \\
15.0 & 2.99 & 2.94 & 0.013 & 0.019 & 1.02 \\
20.0 & 2.99 & 2.99 & 0.017 & 0.014 & 1.00 \\
25.0 & 3.06 & 2.93 & 0.011 & 0.015 & 1.04 \\
\hline \hline
\end{tabular}

impacting drop was analyzed from the image sequences constructed using the images of several identical drop impact experiments taken by changing the time delay of the flash. Liquid drops were ejected from a stainless steel liquid container with an internal diameter of $38 \mathrm{~mm}$ and a length of $60 \mathrm{~mm}$. It comprises a circular orifice of internal and external diameters of 0.4 and $0.6 \mathrm{~mm}$, respectively. The length of the orifice is $8 \mathrm{~mm}$. A contraction is provided in the liquid container just before the orifice entrance. During experiments, the liquid container was closed airtight without any external pressurization and kept at a specified height. Distilled water was used as the experimental liquid whose properties at room temperature are: density $\rho=1000 \mathrm{~kg} / \mathrm{m}^{3}$, surface tension $\sigma=0.073 \mathrm{~N} / \mathrm{m}$, and viscosity $\mu=0.001 \mathrm{~kg} / \mathrm{ms}$. The liquid drops are pinched off by gravity from the orifice exit and arrive at the substrate surface one by one. The size of the drops was measured from their digital video images captured near the substrate surface. For the analysis of experimental results, the drop size $D_{o}$ in a typical drop impact case was taken as the mean diameter value of 15 subsequent drops. Table I shows the mean $\left(D_{o}\right)$ and standard deviation (SD) of several groups of the impacting drops with different impact conditions. The standard deviation was found to be within $2 \%$ of its mean for a majority of the experiments. The sphericity of the drops was estimated in the present study as the ratio of drop diameters measured along the major $(x)$ and minor $(y)$ axes. With the present apparatus, the maximum deviation from sphericity was found to be within 5.8\%. The velocity of an impacting drop $U_{o}$ was estimated as $\sqrt{2 g\left(H-D_{o}\right)}$, where $g$ is the acceleration due to gravity and $H$ is the distance between the orifice exit and the substrate surface. The value of $U_{o}$ was altered by changing $H$. The repeatability for the continuous arrival of the impacting drops at a specific location just above the substrate surface was found to be very high, and hence the variation in $U_{o}$ between the continuously arriving drops was ignored.

The necessary details of the imaging system are given in Fig. 1. An optical triggering device encompassing a light source and a photodiode as light sensor aligned in an axis was employed to generate an electrical signal when a liquid drop cuts through the light passing from the light source to the photodiode. The light source assembly generates a light beam and passes it through a pinhole with a diameter $0.7 \mathrm{~mm}$. The photodiode was placed at a distance of 20-25 $\mathrm{mm}$ from the pinhole. The electric signal from the

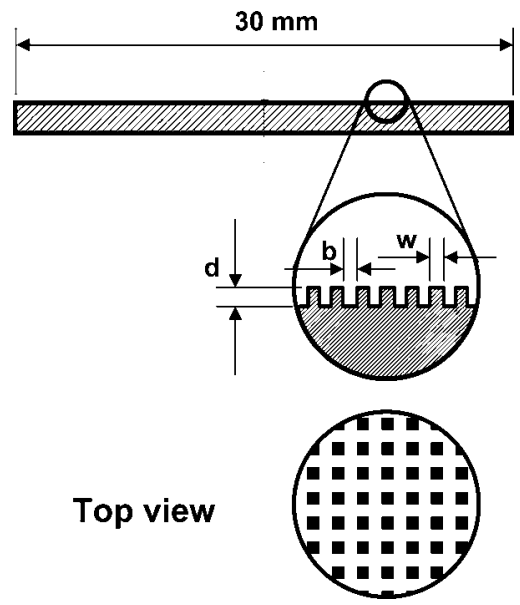

FIG. 2. Details on the geometrical parameters for the textured substrate used in the present study.

photodiode was used to trigger a strobe lamp at a definite instant with the help of a signal delayer. The maximum duration of the light exposure from the strobe lamp is $10 \mu \mathrm{s}$ (as per the product manual), which was sufficient to freeze the motion of the impacting drops for the present experimental conditions. The reflected light from the substrate surface region was captured by a digital video microscope (Keyence VH-5000) and recorded onto a video tape for analysis. In order to retain a high quality of the recorded images, the recordings were done with S-VHS tapes. Later, the images were carefully downloaded to a computer for further analysis.

Textured substrates were prepared by construction of square posts of a uniform height on flat surfaces. It was accomplished by dicing polished stainless-steel surfaces with a diameter of $30 \mathrm{~mm}$. A schematic illustration of the textured

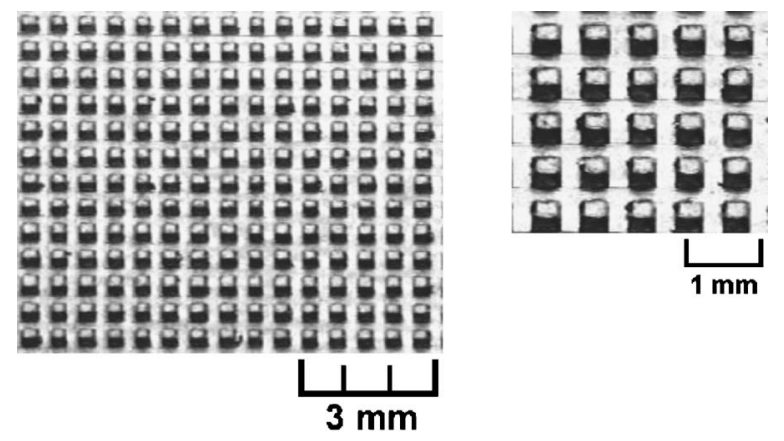

(a)



(b)

FIG. 3. Video images of the textured substrate with $d=b=w=0.3 \mathrm{~mm}(r$ $=2.0$ ). (a) Texture pattern of the substrate and (b) magnified view of the asperities. 
TABLE II. Comparison of the present experimental parameters with those of commercial applications.

\begin{tabular}{lcccccc}
\hline \hline & Substrates & $\begin{array}{c}D_{o} \\
(\mu \mathrm{m})\end{array}$ & We & $\operatorname{Re}$ & $\begin{array}{c}R_{a} \\
(\mu \mathrm{m})\end{array}$ & $R_{a} / D_{o}$ \\
\hline Inkjet & Paper & 50 & $10^{0}-10^{2}$ & $10^{1}-10^{3}$ & $10^{0}-10^{1}$ & $10^{-2}-10^{-1}$ \\
Plasma spraying & Metallic & $30-50$ & $10^{3}-10^{4}$ & $10^{2}-10^{4}$ & $10^{-1}-10^{0}$ & $10^{-3}-10^{-2}$ \\
Solder jet & $\begin{array}{c}\text { Metallic/ } \\
\text { nonmetallic }\end{array}$ & $25-125$ & $10^{0}-10^{1}$ & $10^{0}-10^{2}$ & $10^{-1}-10^{0}$ & $10^{-3}-10^{-2}$ \\
Present study & Metallic & $\approx 3100$ & $40-225$ & $3100-7300$ & $O\left(10^{2}\right)$ & $0.03-0.17$ \\
\hline \hline
\end{tabular}

substrates is given in Fig. 2. The height of the square post $d$ was varied from $0.1-0.5 \mathrm{~mm}$, whereas the width of the square post $w$ and the width of the groove $b$ were kept fixed at $0.3 \mathrm{~mm}$. The images of the asperities of the textured substrate with $d=w=0.3 \mathrm{~mm}$, captured under different camera magnifications, are given in Fig. 3. The roughness factor $r$ of the textured substrate, defined as the ratio of the apparent area (true surface area of the asperities and the grooves) to the projected area of the substrate surface, was estimated from the following relation: $:^{25,27}$

$$
r=1+\frac{4 w d}{(w+b)^{2}}
$$

A high-speed air jet was used to remove the liquid collected inside the grooves of the textured substrate before the start of every experiment. Note that it may not be possible with the present experimental apparatus to impinge the liquid drops onto a specific asperity of the textured substrate repeatedly.

The details of the flow parameters of impacting liquid drops are given in the form of nondimensional numbers We and Reynolds number Re. They are expressed in the present study as

$$
\mathrm{We}=\frac{\rho U_{o}^{2} D_{o}}{\sigma} \text { and } \operatorname{Re}=\frac{\rho U_{o} D_{o}}{\mu} .
$$

The experiments are conducted for $\mathrm{We}, \mathrm{Re}$, and $\mathrm{Oh}$ in the ranges of 35-225, 3100-7300, and 0.0019-0.0022, respectively. Note that the Oh number can be obtained from We and $\mathrm{Re}$ as $\sqrt{\mathrm{We}} / \mathrm{Re}$. Table II shows the comparison of the present experimental parameters with those of commercial applications. Quantitative measurements on the temporal variation of the spreading drop $2 R$ are extracted from the digital analysis of video images.

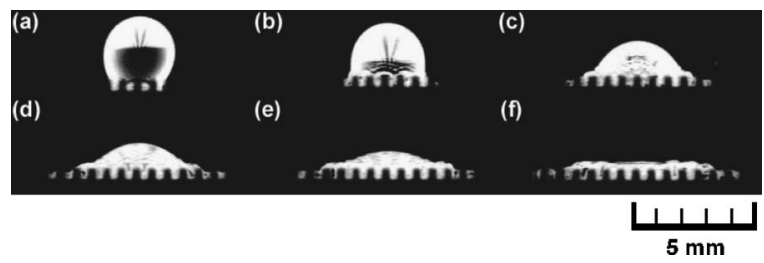

FIG. 4. Spreading process of impacting water drops on the textured substrate with $d=0.3 \mathrm{~mm}(r=2.0)$. $\mathrm{We}=80.2$ and $\mathrm{Oh}=0.00211$. (a) $\tau=0.18$, (b) $\tau=0.32$, (c) $\tau=0.59$, (d) $\tau=0.77$, (e) $\tau=0.96$, and (f) $\tau=1.27$.

\section{RESULTS AND DISCUSSION}

The experimental results obtained from this study are discussed in this section. In order to elucidate the physical role played by the texture geometry, we conducted similar experiments on a smooth stainless-steel surface. Since the impact of liquid drops on smooth solid surfaces has been well reported in literature, we limit our discussion in this paper only to the results obtained with the textured substrates. As in the smooth stainless-steel surface, the spreading liquid wets the textured substrates with the present experimental conditions. However, the contact-angle measurements of the spreading liquid on the textured substrates are not given in this paper. By following the previously published studies, the measurements of $2 R$ are normalized with $D_{o}$, and the time lapse $(t)$ measured from the instant at which the drop touches the top surfaces of the asperities of the textured substrate is nondimensionalized as $\tau=t U_{o} / D_{o}$.

\section{A. Spreading of liquid drops on a textured substrate with asperity height of $0.3 \mathrm{~mm}$}

Figure 4 shows the image sequence for the impact of a water drop with $\mathrm{We}=80.2$ on a stainless-steel textured substrate with $d=0.3 \mathrm{~mm} \quad(r=2.0)$. The rectangular black patches correspond to the asperities. It is observed for the present experimental conditions $(\mathrm{We} \gg 1)$ that the deformation of an impacting drop on the textured substrate at early stages of the impact is qualitatively similar to that on a smooth surface. At later stages, the texture geometry influences the spreading process and develops spreading patterns, which are dramatically different from those observed on the smooth surface.

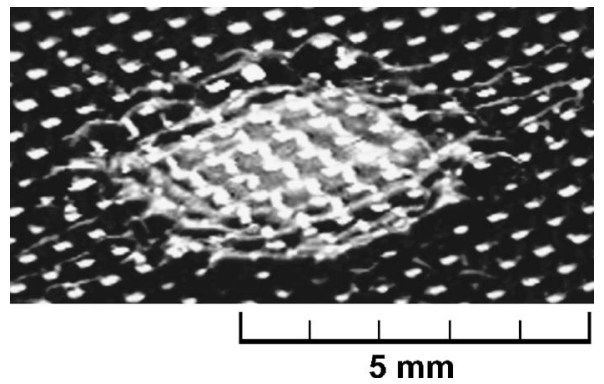

FIG. 5. A video image showing the jet spreading inside the grooves of the textured substrate with $d=0.3 \mathrm{~mm}(r=2.0)$. We $=158.3, \mathrm{Oh}=0.00214$, and $\tau=1.76$. 


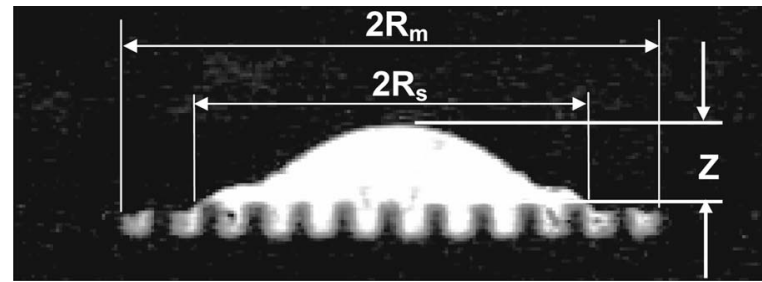

FIG. 6. Sketch illustrating the details of $2 R_{m}, 2 R_{s}$, and $Z$ for the spreading drop.

\section{Two-flow spreading}

It is clear from the images shown in Fig. 4 that the spreading of a liquid drop on a textured substrate is accomplished in two different modes simultaneously: jet spreading, liquid spreading inside the grooves of the textured substrate, and lamella spreading, liquid spreading above the texture pattern of the textured substrate. The images also reveal that the liquid volumes participating in the jet spreading and lamella spreading modes may exhibit different spreading characteristics. A typical video image showing the two spreading modes on the textured substrate is given in Fig. 5. Note that the liquid volume spreading inside the grooves does not fill the grooves entirely but flows like liquid jets in the grooves.

The quantitative measurements of the spreading distance for the jet $\left(2 R_{m}\right)$ and lamella $\left(2 R_{s}\right)$ spreading modes were obtained from the images, similar to those given in Fig. 4. (Refer to Fig. 6 for additional details.) Note that the measurements of $2 R_{m}$ correspond to the maximum width of the spreading liquid inside the grooves for the entire wet region of the textured substrate. In these images, the precise identification of the liquid boundaries in the measurement of $2 R_{m}$ suffers from an error caused by the asperities standing in front of the liquid boundaries. Consequently, the maximum error in $2 R_{m}$ was estimated as of the order $2 w$. This error becomes more significant for the measurements taken during the initial stages of the impact process. The variations of $2 R_{m}$ and $2 R_{s}$ with $\tau$ for the impact of water drops on the textured substrate with $d=0.3 \mathrm{~mm}$ are shown in Fig. 7 for different We's. The measurements of $2 R$ obtained for the impact of water drops on the smooth surface are included in the plots with an intention to make quantitative comparisons on the spreading process.

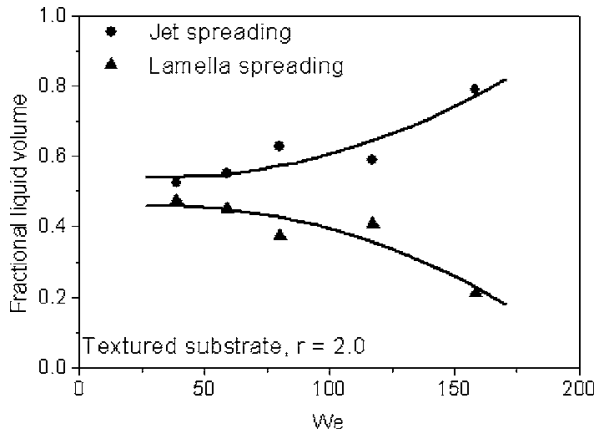

FIG. 8. The influence of We on the variation of the fractional liquid volume entrapped inside the grooves of the textured substrate with $d=0.3 \mathrm{~mm}(r$ $=2.0$ ) after the completion of the impact process.

The variation of the maximum spreading distance in the grooves $\left(2 R_{m}\right)$ deviates considerably from that on the smooth surface $(2 R)$ at low We. As We increases, the variation of $2 R_{m}$ gradually approaches the values of $2 R$. Since the measurements of $2 R_{m}$ were recorded from the type of images shown in Fig. 4, they are identical to the liquid spreading distance in the groove passing through the impact point. This groove resembles a microchannel with periodic openings along its two sides for the spreading liquid. Therefore, in the absence of any influence from the textured substrate, the variation of $2 R_{m}$ is expected to be identical with the variation of $2 R$. Nevertheless, the results shown in Fig. 7 clearly suggest that the asperities, standing on both sides of the groove, influence the spreading process in the groove of low-We drop impacts. In other words, the texture pattern inhibits the spreading process of low-We drop impacts.

In contrast to the variation of $2 R_{m}$, the variation of $2 R_{s}$ deviates from the values measured on the smooth surface at all We. The difference between $2 R_{m}$ and $2 R_{s}$ increases with increasing We. This implies that the liquid volumes participating in the jet and lamella spreading modes flow with different spreading velocities. This may be attributed to the variation in the liquid kinetic energy available for the liquid lamella to spread above the texture pattern with increasing We. Such a sharing of liquid kinetic energy between the jet and lamella spreading modes may be controlled by the texture geometry as well as the flow conditions of the impacting drops. For instance, any increase in the inertial force of the impacting drop increases the amount of liquid volume flow-

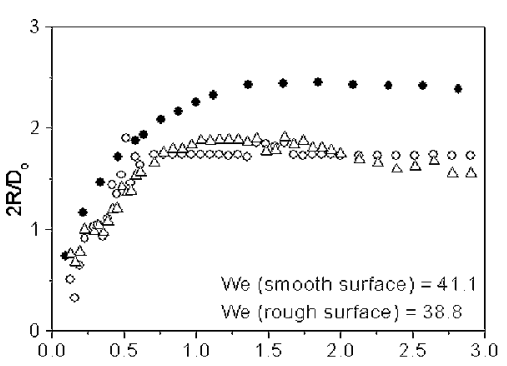

(a)

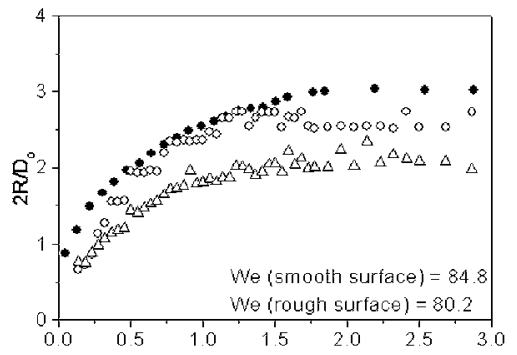

(b)

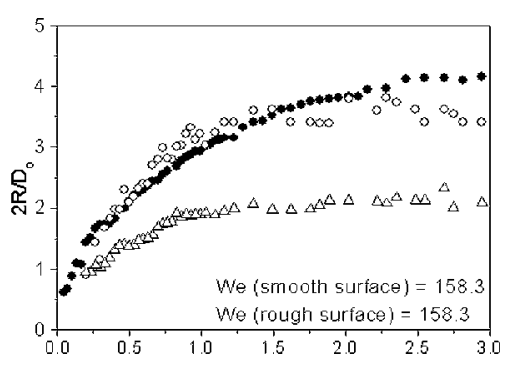

(c)

FIG. 7. The temporal variation of $2 R_{m}$ and $2 R_{s}$ for the impact of liquid drops on the textured substrate with $d=0.3 \mathrm{~mm}(r=2.0)$ during the spreading process with different impact conditions. The open symbols $\left[(\bigcirc) 2 R_{m}\right.$, jet spreading, and $(\triangle) 2 R_{s}$, lamella spreading $]$ correspond to the measurements obtained on the textured substrate. The filled circles correspond to the measurements on the smooth stainless-steel surface. 




FIG. 9. The variation on the maximum spreading drop parameters $\left(2 R_{\max }\right)$ measured on the textured substrate with $d=0.3 \mathrm{~mm}(r=2.0)$ for different We's.

ing inside the grooves. This is shown in Fig. 8, which indicates the effect of We on the variation of the fraction of liquid drop volume resting above the texture pattern of the textured substrate after the completion of the impact process. It was estimated by using the geometrical details, extracted from the corresponding images, of the resting drop above the texture. Note that the shape of the resting drop above the texture is a spherical cap. The remaining liquid volume corresponds to the liquid volume inside the grooves. Since the liquid volume flowing inside the grooves increases with increasing $\mathrm{We}$, the available kinetic energy for the liquid above the texture decreases with increasing We.

The variation on the maximum spreading diameter $2 R_{\max }$ for the impacting drops on the textured substrate with $d$ $=0.3 \mathrm{~mm}(r=2.0)$ is shown in Fig. 9 in terms of We. The values of $2 R_{m \text {, max }}$ are found to be much less than the values measured on the smooth surface in the lower range of We. However, they gradually approach the smooth surface values as We increases. It may be observed from Fig. 9 that the maximum drop diameter measured above the texture pattern of the substrate $\left(2 R_{s, \max }\right)$ is almost independent of We. However, it must be noted that the liquid volume spreading above the textured pattern decreases with increasing We, and therefore the variation of $2 R_{s, \max }$ given in Fig. 9 is something to be expected.

\section{Spreading pattern on the textured substrate}

It is well observed that a liquid drop impacting on a smooth solid surface spreads in an axisymmetric circular pattern. On a textured substrate, the surface geometry influences

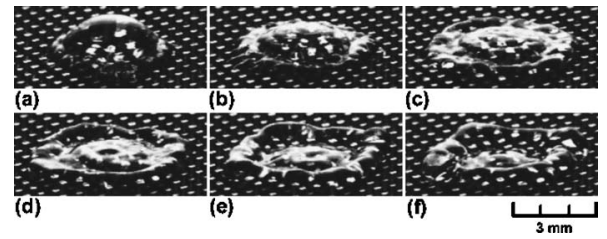

FIG. 10. Spreading process of impacting water drops on the textured substrate with $d=0.3 \mathrm{~mm}(r=2.0)$. The images were captured by placing the camera looking down at an inclined position with an angle of $25^{\circ}-30^{\circ}$ from the horizontal plane. $\mathrm{We}=38.8$ and $\mathrm{Oh}=0.00211$. (a) $\tau=0.65$, (b) $\tau=0.84$, (c) $\tau=1.03$, (d) $\tau=1.23$, (e) $\tau=1.48$, and (f) $\tau=1.74$.

the spreading process and hence the spreading pattern of the impacting drop. Figure 10 shows the sequence of time-lapsed images of the lamella spreading above the texture pattern of the textured substrate with $d=0.3 \mathrm{~mm}(r=2.0)$. The We for the impacting drop is 38.8. In the early stages of impact, the liquid lamella spreads almost in a circular pattern, as seen in Fig. 10 due to the dominance of inertial forces. As the lamella velocity decreases with time, the spreading pattern gradually changes from a circular shape to a rhombus shape. This may be attributed to the surface wetting forces, which become important in the spreading process as the lamella velocity decreases.

Attempts were made to trace the liquid spreading inside the grooves for the entire area of the textured substrate after the completion of the impact process. Figure 11 illustrates the final liquid-covered region, identified by the shaded portion, in the grooves of the textured substrate with $d$ $=0.3 \mathrm{~mm}(r=2.0)$ for different We's. The black squares indicate the asperities. The shaded portion only indicates the region in the grooves at which a contact between the liquid and the solid exists. Note that the asperities entrap air bubbles behind them as seen in Fig. 12 for a typical impact case studied here. The details of such entrapped air bubbles and the liquid-truncated sphere resting above the texture pattern are not shown in the sketches given in Fig. 11. These sketches were prepared by using several segments of the magnified images of the final drop shape captured from the top. A regular spreading pattern of rhombus shape is seen in Fig. 11(a) for the impacting drop with $\mathrm{We}=38.8$. The liquid establishes a corner-to-corner contact between the asperities. The pattern becomes complex as We increases due to the liquid spreading in the grooves. At high $\mathrm{We}$, the grooves

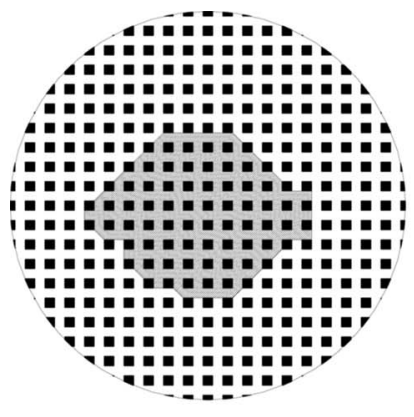

(a)

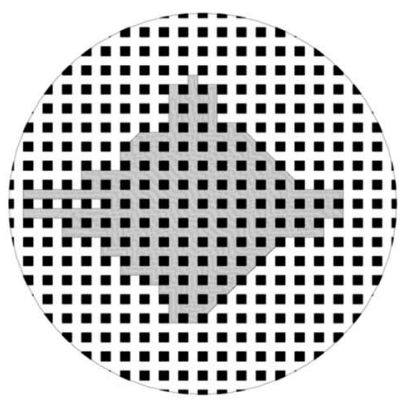

(b)

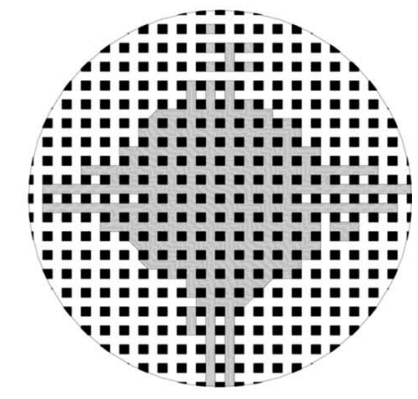

(c)
FIG. 11. Final spreading patterns seen on the textured substrate with $d$ $=0.3 \mathrm{~mm}(r=2.0)$ for different We. (a) $\mathrm{We}=38.8, \quad \mathrm{Oh}=0.00211, \quad$ (b) $\mathrm{We}$ $=80.2, \mathrm{Oh}=0.00211$, and (c) $\mathrm{We}$ $=158.3, \mathrm{Oh}=0.00214$ 


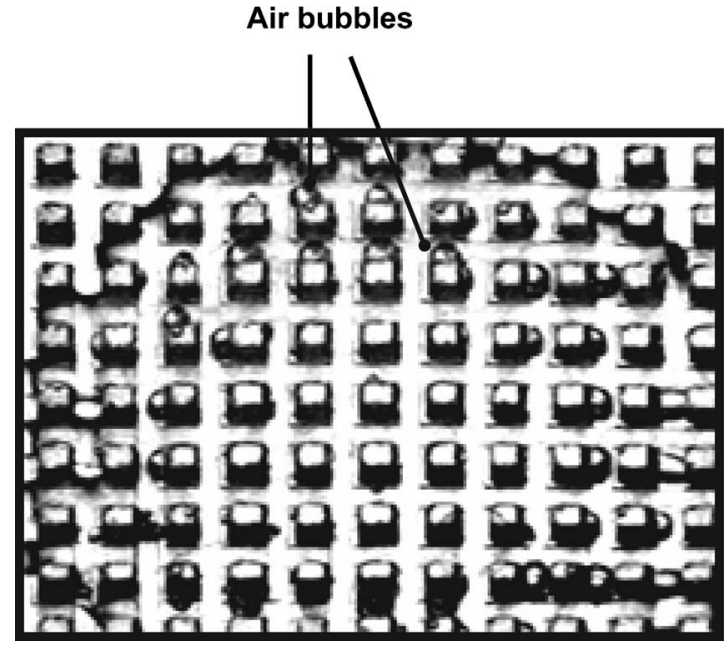

FIG. 12. A digital video image showing the entrapped air bubbles behind the asperities of the textured substrate with $d=0.3 \mathrm{~mm} \quad(r=2.0)$. We $=158.3$ and $\mathrm{Oh}=0.00209$.

force the liquid to spread away from the impact point, forming long liquid-filled grooves on the substrate.

For the textured substrates used in the present study, the arrangement of asperities forms channel-like grooves between any two asperity rows/columns. Figure 13 schematically illustrates an axisymmetric spreading liquid front encountering the asperities of the textured substrate. The velocity of the liquid front in the $x$ direction is maximum in the groove marked " $\mathrm{A}$ " and decreases in other grooves depending on their distance from groove "A." This variation of the liquid velocity in the grooves may be responsible for the rhombus pattern seen in Fig. 11. Note that the liquid flow in the grooves, for example, those marked "A," "B," "C," etc., is identical to the liquid spreading on a smooth surface. But in an asperity row/column (along the line of black squares in Fig. 13), the spreading liquid encounters a significant loss of kinetic energy due to the viscous and surface forces. In other words, the liquid spreading in an asperity row/column reaches a shorter distance compared with that in the adjacent grooves. Since the spreading process is controlled by com-

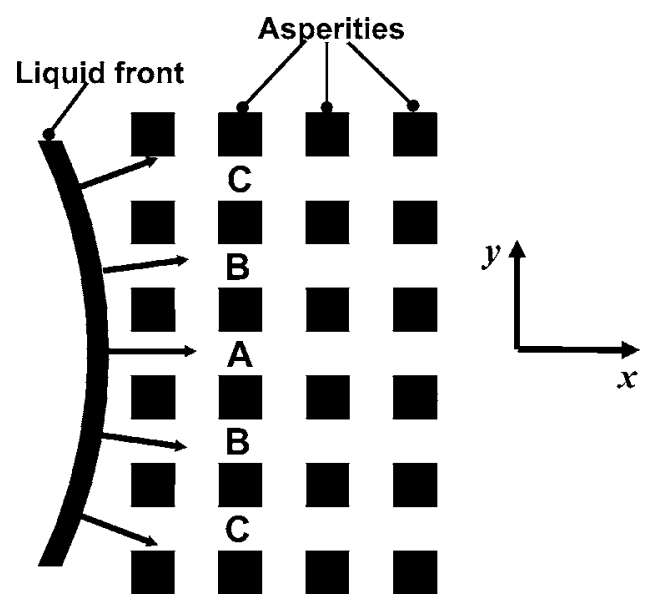

FIG. 13. Schematic sketch showing the velocity vectors (arrow marks) of the spreading liquid front in the grooves of the textured substrate.

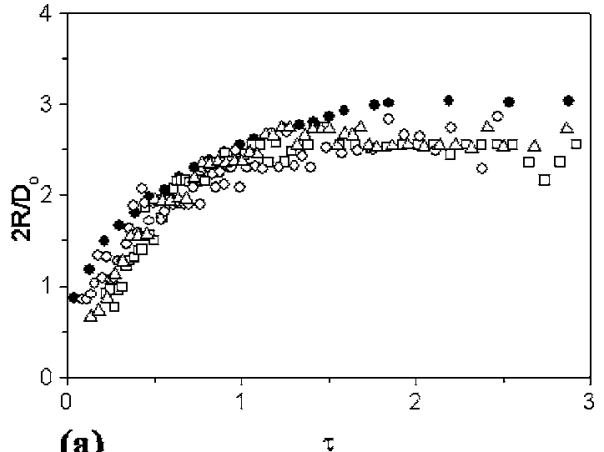

(a)

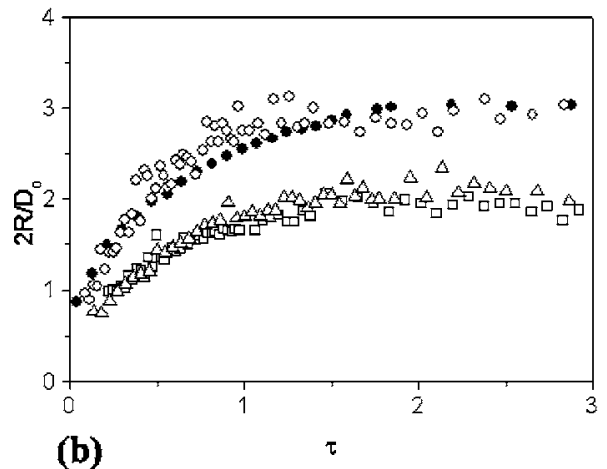

FIG. 14. The temporal variation of $2 R_{m}$ and $2 R_{s}$ for the impact of liquid drops on the textured substrates with varying asperity height $d$. The open symbols correspond to the measurements for: $(\bigcirc) d=0.1 \mathrm{~mm} \quad(r=1.33)$, $\mathrm{We}=78.9, \mathrm{Oh}=0.00211 ;(\triangle) d=0.3 \mathrm{~mm}(r=2.0), \mathrm{We}=80.2, \mathrm{Oh}=0.00211$; and $(\square) d=0.5 \mathrm{~mm}(r=2.67), \mathrm{We}=78.4, \mathrm{Oh}=0.00211$. The filled circles correspond to the measurements on the smooth surface. (a) Jet spreading $2 R_{m}$ and (b) lamella spreading $2 R_{s}$. Note that the measurements of $2 R_{s}$, taken from the similar images as shown in Fig. 15(a), correspond to the width of the continuous portion of white patches seen above the texture, and the curvature effect is ignored in the measurements.

parable contributions of both the surface and inertial forces for the impact of low-We droplets, a distinguishable flow in the grooves may not be observed for low We, thereby forming a regular rhombus as in Fig. 11(a). However, as We increases due to the dominant effect of inertial forces, the liquid in the grooves spreads to farther locations and therefore becomes more distinguishable compared with the liquid spreading in the asperity rows/columns.

\section{B. Spreading of impacting drops on textured substrates with varying asperity heights}

In the interaction between the liquid lamella and the textured substrate, the asperity height is one of the most significant geometrical parameters to influence the impact process. In order to elucidate the effect of asperity height on the spreading process, two additional textured substrates were prepared with $d=0.1$ and $0.5 \mathrm{~mm}(r=1.33$ and 2.67). Similar experiments of drop impact were conducted on the same range of impact conditions as was done for the textured substrate with $d=0.3 \mathrm{~mm}$.

Figure 14 shows the temporal behavior of $2 R_{s}$ and $2 R_{m}$ obtained during the impact of water drops with $\mathrm{We} \approx 80$ on the textured substrates with increasing asperity height. In contrast to the results obtained for the textured substrate with $d=0.3 \mathrm{~mm}(r=2.0)$, the variation of $2 R_{s}$ for the textured sub- 

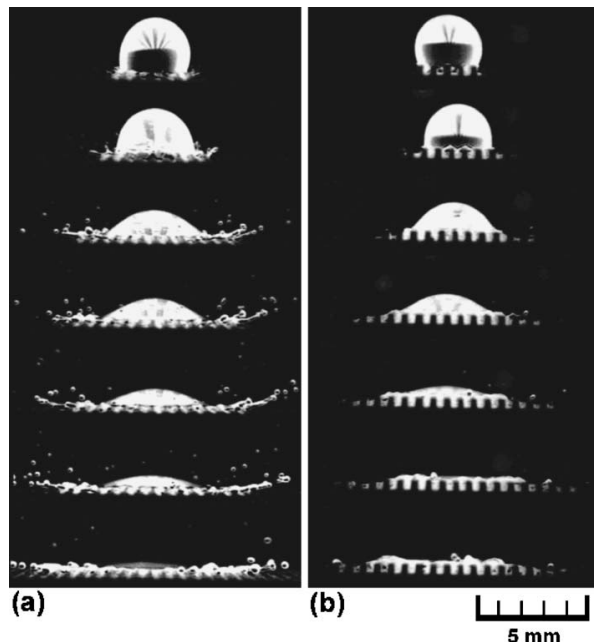

(a)
$5 \mathrm{~mm}$

FIG. 15. Spreading of impacting water drops on the textured substrates with varying asperity height $d$. The numbers given in the parentheses correspond to the value of $\tau$ for the image frames. (a) $d=0.1 \mathrm{~mm}, r=1.33, \mathrm{We}=166.1$, $\mathrm{Oh}=0.00209(0.16,0.32,0.57,0.76,0.91,1.07$, and 1.39) and (b) $d$ $=0.3 \mathrm{~mm}, r=2.0, \mathrm{We}=158.3, \mathrm{Oh}=0.00214(0.20,0.33,0.60,0.76,1.03$, 1.36, and 1.76).

strate with $d=0.1 \mathrm{~mm}(r=1.33)$ follows the trend obtained for the smooth surface. However, the analysis of the digital images reveals that the flow behavior of the lamella spreading on the textured substrate with $d=0.1 \mathrm{~mm}(r=1.33)$ is drastically different from that on the smooth surface. Additional details are given in the next section. For the textured substrates with $d=0.3 \mathrm{~mm}(r=2.0)$ and $d=0.5 \mathrm{~mm}(r$ $=2.67)$, the variation of $2 R_{s}$ and the spreading patterns are similar.

\section{Splashing of impacting drops on textured substrates}

On a dry solid surface, an impacting drop can splash if the inertial force is much larger than the sum of surface and viscous forces. During the splash, tiny liquid droplets detach from the fingers formed at the leading edge of the spreading liquid lamella. Figure 15 shows the time-lapsed image sequences for the impact of water drops on the textured substrates with $d=0.1 \mathrm{~mm}(r=1.33)$ and $d=0.3 \mathrm{~mm}(r=2.0)$. The flow conditions for the two impact cases can be considered as identical for the discussion given below. The images show that the spreading process on the textured substrate with $d=0.1 \mathrm{~mm}(r=1.33)$ features an intense ejection of tiny liquid droplets, and an increase in asperity height of the textured substrate with $d=0.3 \mathrm{~mm}(r=2.0)$ reduces this droplet ejection process drastically. In order to provide additional details of the liquid splashing on the textured substrates, an image sequence obtained with an inclined camera position is given in Fig. 16 for the impact of water drops on the textured substrate with $d=0.1 \mathrm{~mm}(r=1.33)$.

It is observed from Figs. 15(a) and 16 that the liquid front gradually lifts away and develops several thin, long liquid jets from its leading edge. Further analysis shows that these liquid jets eject tiny liquid droplets (refer to Fig. 16), similar to the droplet detachment process that occurs in the breakup of a round liquid jet subjected to capillary

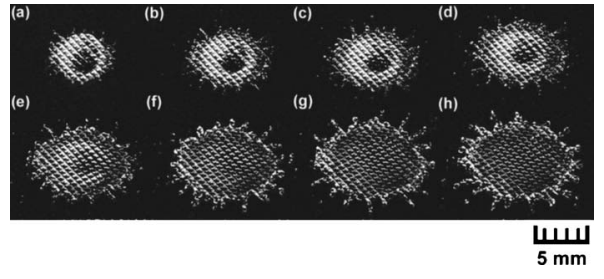

FIG. 16. Liquid splashing of impacting water drops on the textured substrate with $d=0.1 \mathrm{~mm}(r=1.33)$. The images were captured by placing the camera looking down at an inclined position with an angle of $35^{\circ}-45^{\circ}$ from the horizontal plane. $\mathrm{We}=166.1$ and $\mathrm{Oh}=0.00209$. (a) $\tau=0.44$, (b) $\tau=0.57$, (c) $\tau=0.69$, (d) $\tau=0.82$, (e) $\tau=1.07$, (f) $\tau=1.39$, (g) $\tau=1.58$, and (h) $\tau=1.77$. A background elimination process, subtracting the corresponding pixel values of the image taken without the spreading drop (reference image) from the images taken with the spreading drop, was employed in the digital analysis of the images in order to highlight the pattern of thin liquid jets developing from the lamella front.

instability. ${ }^{28}$ Although the liquid jets seen in Fig. 16 resemble the finger-like liquid jets observed during the drop impact process on a smooth surface, their flow characteristics are entirely different from one another as indicated below. For the sake of discussion purposes, an image sequence for the impact of water drops with $\mathrm{We}=158.3$ on the smooth surface is given in Fig. 17.

(1) The liquid jets on the textured substrate develop immediately after the start of the impact process. For instance, the liquid jets and ejected droplets are seen in the images given in Figs. 15 and 16 at instant $\tau<1$. A comparison of image sequences given in Figs. 16 and 17 reveals that the liquid jets may be growing much faster than the fingers observed on the smooth surface. From previously published studies of drop impact on smooth surfaces, it is noted that the liquid droplets detach from the fingers at an instant well beyond $\tau_{\max }{ }^{16}$

(2) For a given We, the number of liquid jets developing from the leading edge of the spreading lamella on the textured substrate is less than that on the smooth surface. For instance, the number of thin liquid jets observed on the textured substrate for the impact case with We $=166.1$, shown in Fig. 16, is around 17-19, whereas the number of perturbations seen in the liquid front on the smooth surface for the similar range of We, shown in Fig. 17, is around 26-32.

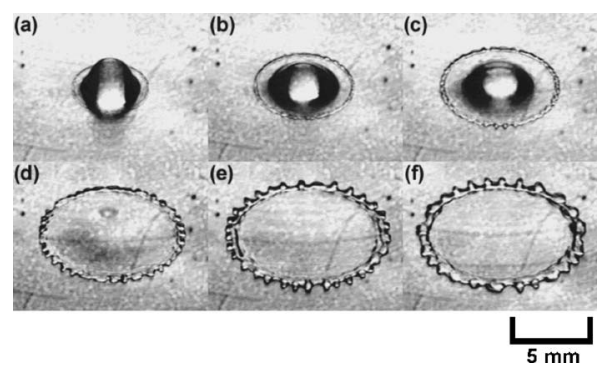

FIG. 17. Spreading of impacting water drops on a smooth stainless-steel surface. The images were captured by placing the camera looking down at an inclined position with an angle of $35^{\circ}-45^{\circ}$ from the horizontal plane. $\mathrm{We}=158.3$ and $\mathrm{Oh}=0.00209$. (a) $\tau=0.36$, (b) $\tau=0.63$, (c) $\tau=0.89$, (d) $\tau$ $=1.42$, (e) $\tau=2.09$, and (f) $\tau=2.55$. 
(3) In contrast to the uniform-sized fingers seen on the smooth surface, the size of liquid jets seen on the textured substrate may not be uniform in length over the entire periphery of the spreading lamella.

The salient features of liquid splashing observed on the textured substrates are in qualitative agreement with the splashing characteristics observed during the impact of drops on rough solid surfaces. The photographs, taken by Range and Feuillebois, ${ }^{15}$ for the analysis of the drop impact process on different rough surface geometries clearly show that the splashing occurs immediately after the start of the impact process. This observation is in line with the features of liquid splashing noted from the present study. A qualitative agreement is also observed in the present study on the decreasing trend of the number of perturbations formed in the lamella with increasing surface roughness. ${ }^{15}$

A simple order-of-magnitude analysis of the thickness of the spreading lamella, based on a previous study of drop impact on a smooth surface, ${ }^{8}$ with the present experimental conditions indicates that the asperities of the textured substrate with $d=0.1 \mathrm{~mm}(r=1.33)$ submerge into the liquid lamella during the spreading process. This is very well observed from the images captured. In other words, a larger portion of the liquid drop volume spreads as liquid lamella above the texture pattern for the textured substrate with a shorter asperity height. Thus for a given drop impact case, the kinetic energy available for the liquid lamella to spread above the texture pattern increases with decreasing asperity height. Since the intensity of splashing increases with increasing liquid kinetic energy, the liquid lamella spreading in the textured substrate $d=0.1 \mathrm{~mm}(r=1.33)$ exhibits a more intense splashing.

Previous analyses of the drop impact process suggest that the physical mechanism behind the formation of liquid fingers in the liquid lamella spreading on a smooth surface is Rayleigh-Taylor instability, caused by the deceleration of the liquid-air interface. ${ }^{29-31}$ By following this instability mechanism, Bussmann et al. ${ }^{11}$ reported numerical simulations of the drop impact on rough surfaces by adjusting the instability growth parameter. Nevertheless, an explicit relationship between the instability growth parameter and the roughness characteristics is not yet available. Although the RayleighTaylor instability may still be valid for the liquid lamella spreading over the texture pattern, the physical mechanisms involved in the formation of thin liquid jets and tiny droplets, seen on the images of Figs. 15 and 16 could be different from this instability. Our judgment here is fully based on the above-mentioned flow features, listed (1) to (3). The available data shows that the texture geometry plays a significant role in controlling the growth of thin liquid jets formed in the lamella front. However, a detailed experimental study is necessary to further understand this observation.

\section{CONCLUSIONS}

This experimental investigation has consolidated the salient features involved in the spreading process of impacting water drops with varying impact conditions on structured rough substrates. The major conclusions are the following.

(1) On structured rough substrates (textured substrates), an impacting liquid drop spreads simultaneously both inside the grooves and above the texture pattern of the substrate. For the impact of high-We drops, the liquid flowing inside the grooves of the textured substrate (jet spreading) dominates the spreading process. In contrast, the liquid flowing above the texture pattern (lamella spreading) dominates for the impact of low-We drops.

(2) In contrast to the circular spreading pattern seen on a smooth surface, the spreading pattern on a textured substrate resembles the shape of a rhombus, mainly caused by the arrangement and orientation of the surface asperities. A regular rhombus shape is observed for the impact of low-We drops, and the shape becomes complex at high We.

(3) The liquid spreading inside the grooves reaches the same distance as on the smooth surface for the impact of high-We drops. The spreading diameter measured for the liquid volume flowing above the texture pattern of the substrate is smaller than that on the smooth surface, mainly attributable to the decrease in the liquid kinetic energy available above the texture pattern. For a given droplet We, the texture geometry determines the liquid kinetic energy available above the substrate.

(4) The texture pattern of the substrate may disturb the liquid lamella, and it makes the liquid splash by developing liquid jets from the liquid front. It is observed qualitatively that these liquid jets may be exhibiting different characteristics from the liquid fingers seen on the smooth surface.

(5) The effect of the asperity height of the textured substrates on the drop impact process is more pronounced if the asperity height is comparable with the thickness of the liquid lamella developing from the impact.

\section{ACKNOWLEDGMENT}

This research has been partly supported by the Japan Society for the Promotion of Science (JSPS) during the academic year 2004.

${ }^{1}$ S. E. Bechtel, D. B. Bogy, and F. E. Talke, "Impact of liquid drop against a flat surface," IBM J. Res. Dev. 25, 963 (1981).

${ }^{2}$ F. Gao and A. A. Sonin, "Precise deposition of molten micro-drops: The physics of digital microfabrication," Proc. R. Soc. London, Ser. A 444, 533 (1994).

${ }^{3}$ C. H. Amon, K. S. Schmaltz, R. Merz, and F. B. Prinz, "Numerical and experimental investigation of interface bonding via substrate remelting of an impinging molten metal droplet," ASME Trans. J. Heat Transfer 118, 164 (1996).

${ }^{4}$ D. J. Hayes and D. B. Wallace, "Solder jet printing: Wafer bumping and CSP applications," Chip Scale Rev. 2, 75 (1998).

${ }^{5}$ M. F. Orme and R. F. Smith, "Enhanced aluminum properties by means of precise droplet deposition,” ASME J. Manuf. Sci. Eng. 122, 484 (2000).

${ }^{6}$ B. Y. Tay and M. J. Edirisinghe, "Investigation of some phenomena occurring during continuous ink-jet printing of ceramics," J. Mater. Res. 16, 373 (2001).

${ }^{7}$ M. Rein, "Phenomena of liquid drop impact on solid and liquid surfaces," Fluid Dyn. Res. 12, 61 (1993). 
${ }^{8}$ J. Fukai, Y. Shiba, T. Yumamoto, O. Miyatake, D. Poulikakos, C. M. Megaridis, and Z. Zhao, "Wetting effects on the spreading of a liquid droplet with a flat surface: Experiment and modeling," Phys. Fluids 7, 236 (1995).

${ }^{9}$ M. Pasandideh-Fard, S. Qiao, S. Chandra, and J. Mostaghimi, "Capillary effects during droplet impact on a solid surface," Phys. Fluids 8, 650 (1996).

${ }^{10}$ S. Schiaffino and A. A. Sonin, "Molten droplet deposition and solidification at low Weber numbers," Phys. Fluids 9, 3172 (1997).

${ }^{11}$ M. Bussmann, S. Chandra, and J. Mostaghimi, "Modeling the splash of a droplet impacting a solid surface," Phys. Fluids 12, 3121 (2000).

${ }^{12}$ H.-Y. Kim and J.-H. Chun, "The recoiling of liquid droplets upon collision with solid surfaces," Phys. Fluids 13, 643 (2001).

${ }^{13}$ I. V. Roisman, R. Rioboo, and C. Tropea, "Normal impact of a liquid droplet on a dry surface: Model for spreading and receding," Proc. R. Soc. London, Ser. A 458, 1411 (2002).

${ }^{14}$ C. D. Stow and M. G. Hadfield, "An experimental investigation of fluid flow resulting from the impact of a water drop with an unyielding dry surface," Proc. R. Soc. London, Ser. A 373, 419 (1981).

${ }^{15} \mathrm{~K}$. Range and F. Feuillebois, "Influence of surface roughness on liquid droplet impact," J. Colloid Interface Sci. 203, 16 (1998).

${ }^{16} \mathrm{~S}$. Shakeri and S. Chandra, "Splashing of molten tin droplets on a rough steel surface," Int. J. Heat Mass Transfer 45, 4561 (2002).

${ }^{17}$ C. Mundo, M. Sommerfield, and C. Tropea, "Droplet-wall collisions: Experimental studies of the deformation and breakup process," Int. J. Multiphase Flow 21, 151 (1995).

${ }^{18} \mathrm{H}$. Marmanis and S. T. Thoroddsen, "Scaling of the fingering pattern of an impacting drop," Phys. Fluids 8, 1344 (1996).
${ }^{19}$ R. Rioboo, M. Marengo, and C. Tropea, "Time evolution of liquid drop impact onto solid, dry surfaces," Exp. Fluids 33, 112 (2002).

${ }^{20}$ P. G. de Gennes, "Wetting: Statics and dynamics," Rev. Mod. Phys. 57, 827 (1985).

${ }^{21}$ L. M. Hocking, "A moving fluid interface on a rough surface," J. Fluid Mech. 76, 801 (1976).

${ }^{22}$ G. Wolansky and A. Marmur, "Apparent contact angles on rough surfaces: The Wenzel equation revisited," Colloids Surf., A 156, 381 (1999).

${ }^{23}$ S. Herminghaus, "Roughness-induced non-wetting," Europhys. Lett. 52, 165 (2000).

${ }^{24}$ D. Öner and T. McCarthy, "Ultrahydrophobic surfaces. Effects of topography length scales on wettability," Langmuir 16, 7777 (2000).

${ }^{25}$ J. Bico, C. Tordeux, and D. Quéré, "Rough wetting," Europhys. Lett. 55, 214 (2001).

${ }^{26}$ J. Bico, U. Thiele, and D. Quéré, "Wetting of textured surfaces," Colloids Surf., A 206, 41 (2002).

${ }^{27}$ Z. Yoshimitsu, A. Nakajima, T. Watanabe, and K. Hashimoto, "Effects of surface structure on the hydrophobicity and sliding behavior of water droplets," Langmuir 18, 5818 (2002).

${ }^{28}$ L. Rayleigh, "On the instability of a cylinder of viscous liquid under capillary force," Philos. Mag. 34, 145 (1892).

${ }^{29}$ R. F. Allen, "The role of surface tension in splashing," J. Colloid Interface Sci. 51, 350 (1975).

${ }^{30}$ H.-Y. Kim, Z. C. Feng, and J.-H. Chun, "Instability of a liquid jet emerging from a droplet upon collision with a solid surface," Phys. Fluids 12, 531 (2000).

${ }^{31}$ S. T. Thoroddsen and J. Sakakibara, "Evolution of the fingering pattern of an impacting drop," Phys. Fluids 10, 1359 (1998). 